\title{
A Unique Common Fixed Point Theorem Using $\psi-\phi$ Condition in a Partial Metric Space Using an ICS Mapping
}

\author{
K.P.R.Rao ${ }^{1}$, G.N.V.Kishore ${ }^{2, *}$, P.R.Sobhana Babu ${ }^{3}$ \\ ${ }^{1}$ Department of Mathematics, Acharya Nagarjuna University,Nagarjuna Nagar, \\ Guntur - 522 510, Andhra Pradesh, India \\ ${ }^{2}$ Department of Mathematics, Baba Institute of Technology and Sciences,P.M.Palem, \\ Madhurawada Visakhapatnam - 530048, Andhra Pradesh, India \\ ${ }^{3}$ Department of Mathematics, Ramachandra College of Engineering, Vatluru(V), \\ Eluru-534007, West Godavari Dist., Andhra Pradesh, India \\ *Corresponding Author: kishore.apr2@gmail.com
}

Copyright @2013 Horizon Research Publishing All rights reserved.

\begin{abstract}
The ICS mapping was introduced by K.P.Chi [On a fixed point theorem for certain class of maps satisfying a contractive condition depended on an another function, Lobachevskii J. math., 30(4), 2009, 289 - 291.] In this paper, we obtain a unique common fixed point theorem in partial metric spaces by using ICS mapping and also introduced supported example to our main theorem.
\end{abstract}

Keywords Partial Metric, ICS Mapping, Complete Space

Mathematics Subject Classification (2000): 54H25, 47H10, 54E50

\section{Introduction and Preliminaries}

The notion of partial metric space was introduced by Matthews [10] as a part of the study of denotational semantics of data flow networks. In fact, it is widely recognized that partial metric spaces play an important role in constructing models in the theory of computation and domain theory in computer science (see e.g. [12, 13, 14, $15,16,17,18,19,20])$.

Matthews [10, 11], Oltra and Valero [23] and Romaguera [21] and Altun et al. [2] proved some fixed point theorems in partial metric spaces for a single map (see also $[1,6,7,8,9,3,4,22,17]$ ).

First we recall some basic definitions and lemmas which play crucial role in the theory of partial metric spaces.

Definition 1.1 (See $[10,11]$ ) A partial metric on a nonempty set $X$ is a function $p: X \times X \rightarrow R^{+}$such that for all $x, y, z \in X$ :

$\left(p_{1}\right) x=y \Leftrightarrow p(x, x)=p(x, y)=p(y, y)$,

$\left(p_{2}\right) p(x, x) \leq p(x, y), p(y, y) \leq p(x, y)$,

$\left(p_{3}\right) p(x, y)=p(y, x)$

$\left(p_{4}\right) p(x, y) \leq p(x, z)+p(z, y)-p(z, z)$.

The pair $(X, p)$ is called a partial metric space (PMS).

Clearly $p(x, y)=0$ implies $x=y$ and $x \neq y$ implies $p(x, y)>0$.

If $p$ is a partial metric on $X$, then the function $d_{p}: X \times X \rightarrow \mathbb{R}^{+}$given by $d_{p}(x, y)=2 p(x, y)-p(x, x)-p(y, y)$ is a metric on $X$. 
Example 1.2 (See e.g. $[11,7,1])$ Consider $X=[0, \infty)$ with $p(x, y)=\max \{x, y\}$. Then $(X, p)$ is a partial metric space. It is clear that $p$ is not a (usual) metric. Note that in this case $d_{p}(x, y)=|x-y|$.

Each partial metric $p$ on $X$ generates a $T_{0}$ topology $\tau_{p}$ on $X$ which has as a base the family of open $p$-balls $\left\{B_{p}(x, \varepsilon), x \in X, \varepsilon>0\right\}$, where $B_{p}(x, \varepsilon)=\{y \in X: p(x, y)<p(x, x)+\varepsilon\}$ for all $x \in X$ and $\varepsilon>0$.

We now state some basic topological notions (such as convergence, completeness, continuity) on partial metric spaces (see e.g. $[10,11,2,1,7,9]$.)

\section{Definition 1.3}

1. A sequence $\left\{x_{n}\right\}$ in the PMS $(X, p)$ converges to the limit $x$ if and only if $p(x, x)=\lim _{n \rightarrow \infty} p\left(x, x_{n}\right)$.

2. A sequence $\left\{x_{n}\right\}$ in the PMS $(X, p)$ is called a Cauchy sequence if $\lim _{n, m \rightarrow \infty} p\left(x_{n}, x_{m}\right)$ exists and is finite.

3. A PMS $(X, p)$ is called complete if every Cauchy sequence $\left\{x_{n}\right\}$ in $X$ converges with respect to $\tau_{p}$, to a point $x \in X$ such that $p(x, x)=\lim _{n, m \rightarrow \infty} p\left(x_{n}, x_{m}\right)$.

4. A mapping $F: X \rightarrow X$ is said to be continuous at $x_{0} \in X$ if for every $\epsilon>0$, there exists $\delta>0$ such that $F\left(B_{p}\left(x_{0}, \delta\right)\right) \subseteq B_{p}\left(F x_{0}, \epsilon\right)$

We need the following lemmas in $\operatorname{PMS}([10,11,1,2,7,9])$.

\section{Lemma 1.4}

1. A sequence $\left\{x_{n}\right\}$ is a Cauchy sequence in the PMS $(X, p)$ if and only if it is a Cauchy sequence in the metric $\operatorname{space}\left(X, d_{p}\right)$.

2. A PMS $(X, p)$ is complete if and only if the metric space $\left(X, d_{p}\right)$ is complete. Moreover

$$
\lim _{n \rightarrow \infty} d_{p}\left(x, x_{n}\right)=0 \Leftrightarrow p(x, x)=\lim _{n \rightarrow \infty} p\left(x, x_{n}\right)=\lim _{n, m \rightarrow \infty} p\left(x_{n}, x_{m}\right)
$$

Lemma 1.5 Assume $x_{n} \rightarrow z$ as $n \rightarrow \infty$ in a PMS $(X, p)$ such that $p(z, z)=0$. Then $\lim _{n \rightarrow \infty} p\left(x_{n}, y\right)=p(z, y)$ for every $y \in X$.

In this paper, we obtain a unique common fixed point theorem for two mappings using ICS mapping in Partial metric spaces. Our result generalizes the recent several known results.

Recently [5] introduced the concept of ICS mapping as follows.

Definition 1.6 [5] Let $(X, d)$ be a metric space. A mapping $T: X \rightarrow X$ is said to be ICS if $T$ is injective, continuous and has the property : for every sequence $\left\{x_{n}\right\}$ in $X$, if $\left\{T x_{n}\right\}$ is convergent then $\left\{x_{n}\right\}$ is also convergent.

\section{MAIN RESULT}

Let $\Psi$ denote the set of all continuous and monotonically increasing functions $\psi:[0, \infty) \rightarrow[0, \infty)$.

Let $\Phi$ denote the set of all lower semi continuous functions $\phi:[0, \infty) \rightarrow[0, \infty)$ such that $\phi(t)>0$ for $t>0$.

Theorem 2.1 Let $(X, p)$ be a partial metric space and $T: X \rightarrow X$ be an ICS mapping and $F, G: X \rightarrow X$ be satisfying

$$
\begin{aligned}
\psi(p(T F x, T G y)) \leq \psi & \left(\max \left\{\begin{array}{c}
p(T x, T y), p(T x, T F x), p(T y, T G y), \\
\frac{1}{2}[p(T x, T G y)+p(T y, T F x)]
\end{array}\right\}\right) \\
& -\phi\left(\max \left\{\begin{array}{c}
p(T x, T y), p(T x, T F x), p(T y, T G y), \\
\frac{1}{2}[p(T x, T G y)+p(T y, T F x)]
\end{array}\right\}\right),
\end{aligned}
$$

$\forall x, y \in X$, where $\psi \in \Psi$ and $\phi \in \Phi$. Then $F$ and $G$ have a unique common fixed point in $X$.

Let $x_{0} \in X$. Define $x_{2 n+1}=F x_{2 n}, x_{2 n+2}=G x_{2 n+1}, n=0,1,2, \cdots$ and $y_{n}=T x_{n}, n=0,1,2, \cdots$

Case(a): Suppose $y_{2 n+1}=y_{2 n}$ for some $n$.

Then $T x_{2 n+1}=T x_{2 n}$.

Since $T$ is injective, we have $x_{2 n+1}=x_{2 n} \Rightarrow F x_{2 n}=x_{2 n}$. 
Thus $F \alpha=\alpha$, where $\alpha=x_{2 n}$.

Suppose $T \alpha \neq T G \alpha$. Consider

$$
\begin{aligned}
\psi(p(T \alpha, T G \alpha))= & \psi(p(T F \alpha, T G \alpha)) \\
\leq & \psi\left(\max \left\{\begin{array}{c}
p(T \alpha, T \alpha), p(T \alpha, T F \alpha), p(T \alpha, T G \alpha), \\
\frac{1}{2}[p(T \alpha, T G \alpha)+p(T \alpha, T F \alpha)]
\end{array}\right\}\right) \\
& -\phi\left(\max \left\{\begin{array}{c}
p(T \alpha, T \alpha), p(T \alpha, T F \alpha), p(T \alpha, T G \alpha), \\
\frac{1}{2}[p(T \alpha, T G \alpha)+p(T \alpha, T F \alpha)]
\end{array}\right\}\right) \\
= & \psi(p(T \alpha, T G \alpha))-\phi(p(T \alpha, T G \alpha)), \text { from }\left(p_{2}\right) \\
< & \psi(p(T \alpha, T G \alpha)),
\end{aligned}
$$

a contradiction.

Hence $T \alpha=T G \alpha$. Since $T$ is injective, we have $\alpha=G \alpha$.

Thus $\alpha$ is a common fixed point of $F$ and $G$.

If $\beta$ is another common fixed point of $F$ and $G$, then $T \alpha \neq T \beta$.

$$
\begin{aligned}
\psi(p(T \alpha, T \beta))= & \psi(p(T F \alpha, T G \beta)) \\
\leq & \psi\left(\max \left\{\begin{array}{c}
p(T \alpha, T \beta), p(T \alpha, T F \alpha), p(T \beta, T G \beta), \\
\frac{1}{2}[p(T \alpha, T G \beta)+p(T \beta, T F \alpha)]
\end{array}\right\}\right) \\
& -\phi\left(\max \left\{\begin{array}{c}
p(T \alpha, T \beta), p(T \alpha, T F \alpha), p(T \beta, T G \beta), \\
\frac{1}{2}[p(T \alpha, T G \beta)+p(T \beta, T F \alpha)]
\end{array}\right\}\right) \\
= & \psi\left(\max \left\{\begin{array}{c}
p(T \alpha, T \beta), p(T \alpha, T \alpha), p(T \beta, T \beta), \\
\frac{1}{2}[p(T \alpha, T \beta)+p(T \beta, T \alpha)]
\end{array}\right\}\right) \\
& -\phi\left(\max \left\{\begin{array}{c}
p(T \alpha, T \beta), p(T \alpha, T \alpha), p(T \beta, T \beta), \\
\frac{1}{2}[p(T \alpha, T \beta)+p(T \beta, T \alpha)]
\end{array}\right\}\right) \\
= & \psi(p(T \alpha, T \beta))-\phi(p(T \alpha, T \beta)), \text { from }\left(p_{2}\right) \\
< & \psi(p(T \alpha, T G \alpha)),
\end{aligned}
$$

a contradiction.

Thus $\alpha$ is the unique common fixed point of $F$ and $G$.

Case(b) : Assume that $y_{n} \neq y_{n+1}$ for all $n$.

Denote $p_{n}=p\left(y_{n}, y_{n+1}\right)$.

$$
\begin{aligned}
\psi\left(p_{2 n}\right)= & \psi\left(p\left(y_{2 n}, y_{2 n+1}\right)\right) \\
= & \psi\left(p\left(T x_{2 n+1}, T x_{2 n}\right)\right) \\
= & \psi\left(p\left(T F x_{2 n}, T G x_{2 n-1}\right)\right) \\
\leq & \psi\left(\left\{\begin{array}{c}
p\left(y_{2 n}, y_{2 n-1}\right), p\left(y_{2 n}, y_{2 n+1}\right), p\left(y_{2 n-1}, y_{2 n}\right), \\
\frac{1}{2}\left[p\left(y_{2 n}, y_{2 n}\right)+p\left(y_{2 n-1}, y_{2 n+1}\right)\right]
\end{array}\right\}\right) \\
& -\phi\left(\left\{\begin{array}{c}
p\left(y_{2 n}, y_{2 n-1}\right), p\left(y_{2 n}, y_{2 n+1}\right), p\left(y_{2 n-1}, y_{2 n}\right), \\
\frac{1}{2}\left[p\left(y_{2 n}, y_{2 n}\right)+p\left(y_{2 n-1}, y_{2 n+1}\right)\right]
\end{array}\right\}\right) \\
= & \psi\left(\max p_{2 n-1}, p_{2 n}\right)-\phi\left(\max p_{2 n-1}, p_{2 n}\right), \text { from }\left(p_{4}\right)
\end{aligned}
$$

If $p_{2 n}$ is maximum, then $\psi\left(p_{2 n}\right) \leq \psi\left(p_{2 n}\right)-\phi\left(p_{2 n}\right)<\psi\left(p_{2 n}\right)$, a contradiction.

Hence

$$
\begin{aligned}
\psi\left(p_{2 n}\right) & \leq \psi\left(p_{2 n-1}\right)-\phi\left(p_{2 n-1}\right) \\
& <\psi\left(p_{2 n-1}\right)
\end{aligned}
$$

$p_{2 n}<p_{2 n-1}$, since $\psi$ is monotonically non - decreasing.

Similarly, we can show that $p_{2 n+1}<p_{2 n}$. Thus $\left\{p_{n}\right\}$ is monotonically decreasing sequence of non negative real numbers and hence $\left\{p_{n}\right\}$ converges to some $r \geq 0$. Suppose $r>0$.

Letting $n \rightarrow \infty$ in (2.1), we get

$$
\psi(r) \leq \psi(r)-\phi(r)<\psi(r), \text { since } \phi(t)>0 \text { for } t>0 .
$$

It is a contradiction. Hence $r=0$.

Thus

$$
\lim _{n \rightarrow \infty} p\left(y_{n}, y_{n+1}\right)=0 \text {. }
$$

Hence from $\left(p_{2}\right)$, we get

$$
\lim _{n \rightarrow \infty} p\left(y_{n}, y_{n}\right)=0
$$


From the definition of $d_{p}$, using (2.2) and(2.3), we get

$$
\lim _{n \rightarrow \infty} d_{p}\left(y_{n}, y_{n+1}\right)=0 .
$$

Now we prove that $\left\{y_{n}\right\}$ is a Cauchy sequence in $\left(X, d_{p}\right)$.

On contrary suppose that $\left\{y_{2 n}\right\}$ is not Cauchy.

Then there exist an $\epsilon>0$ and monotone increasing sequences of natural numbers $\left\{2 m_{k}\right\}$ and $\left\{2 n_{k}\right\}$ such that $n_{k}>m_{k}$,

$$
d_{p}\left(y_{2 m_{k}}, y_{2 n_{k}}\right) \geq \epsilon
$$

and

$$
d_{p}\left(y_{2 m_{k}}, y_{2 n_{k}-2}\right)<\epsilon
$$

From (2.5) and (2.6), we obtain

$$
\begin{aligned}
\epsilon & \leq d_{p}\left(y_{2 m_{k}}, y_{2 n_{k}}\right) \\
& \leq d_{p}\left(y_{2 m_{k}}, y_{2 n_{k}-2}\right)+d_{p}\left(y_{2 n_{k}-2}, y_{2 n_{k}-1}\right)+d_{p}\left(y_{2 n_{k}-1}, y_{2 n_{k}}\right) \\
& <\epsilon+d_{p}\left(y_{2 n_{k}-2}, y_{2 n_{k}-1}\right)+d_{p}\left(y_{2 n_{k}-1}, y_{2 n_{k}}\right) .
\end{aligned}
$$

Letting $k \rightarrow \infty$ and then using (2.4), we get

$$
\lim _{k \rightarrow \infty} d_{p}\left(y_{2 m_{k}}, y_{2 n_{k}}\right)=\epsilon
$$

Hence from definition of $d_{p}$ and (2.3), we have

$$
\lim _{k \rightarrow \infty} p\left(y_{2 m_{k}}, y_{2 n_{k}}\right)=\frac{\epsilon}{2}
$$

Letting $k \rightarrow \infty$ and then using (2.7) and (2.4) in

$\left|d_{p}\left(y_{2 n_{k}+1}, y_{2 m_{k}}\right)-d_{p}\left(y_{2 n_{k}}, y_{2 m_{k}}\right)\right| \leq d_{p}\left(y_{2 n_{k}+1}, y_{2 n_{k}}\right)$

we obtain

$$
\lim _{k \rightarrow \infty} d_{p}\left(y_{2 n_{k}+1}, y_{2 m_{k}}\right)=\epsilon
$$

Hence, we have

$$
\lim _{k \rightarrow \infty} p\left(y_{2 n_{k}+1}, y_{2 m_{k}}\right)=\frac{\epsilon}{2}
$$

Letting $k \rightarrow \infty$ and then using (2.7) and (2.4) in $\left|d_{p}\left(y_{2 n_{k}}, y_{2 m_{k}-1}\right)-d_{p}\left(y_{2 n_{k}}, y_{2 m_{k}}\right)\right| \leq d_{p}\left(y_{2 m_{k}-1}, y_{2 m_{k}}\right)$, we get

$$
\lim _{k \rightarrow \infty} d_{p}\left(y_{2 n_{k}}, y_{2 m_{k}-1}\right)=\epsilon
$$

Hence, we have

$$
\lim _{k \rightarrow \infty} p\left(y_{2 n_{k}}, y_{2 m_{k}-1}\right)=\frac{\epsilon}{2} .
$$

Letting $k \rightarrow \infty$ and then using (2.11) and (2.4) in

$\left|d_{p}\left(y_{2 m_{k}-1}, y_{2 n_{k}+1}\right)-d_{p}\left(y_{2 m_{k}-1}, y_{2 n_{k}}\right)\right| \leq d_{p}\left(y_{2 n_{k}+1}, y_{2 n_{k}}\right)$

we obtain

$$
\lim _{k \rightarrow \infty} d_{p}\left(y_{2 m_{k}-1}, y_{2 n_{k}+1}\right)=\epsilon
$$

Hence, we get

$$
\lim _{k \rightarrow \infty} p\left(y_{2 m_{k}-1}, y_{2 n_{k}+1}\right)=\frac{\epsilon}{2}
$$

Now,

$$
\begin{aligned}
\psi\left(p\left(y_{2 n_{k}+1}, y_{2 m_{k}}\right)\right)= & \psi\left(p\left(T F x_{2 n_{k}}, T G x_{2 m_{k}-1}\right)\right) \\
\leq & \psi\left(\max \left\{\begin{array}{c}
p\left(y_{2 n_{k}}, y_{2 m_{k}-1}\right), p\left(y_{2 n_{k}}, y_{2 n_{k}+1}\right), \\
p\left(y_{2 m_{k}-1}, y_{2 m_{k}}\right), \\
\frac{1}{2}\left[p\left(y_{2 n_{k}}, y_{2 m_{k}}\right)+p\left(y_{2 m_{k}-1}, y_{2 n_{k}+1}\right)\right]
\end{array}\right\}\right) \\
& -\phi\left(\max \left\{\begin{array}{c}
p\left(y_{2 n_{k}}, y_{2 m_{k}-1}\right), p\left(y_{2 n_{k}}, y_{2 n_{k}+1}\right), \\
p\left(y_{2 m_{k}-1}, y_{2 m_{k}}\right), \\
\frac{1}{2}\left[p\left(y_{2 n_{k}}, y_{2 m_{k}}\right)+p\left(y_{2 m_{k}-1}, y_{2 n_{k}+1}\right)\right]
\end{array}\right\}\right) .
\end{aligned}
$$


Letting $k \rightarrow \infty$ and then using (2.10), (2.12), (2.2), (2.14) and (2.8), we get

$$
\begin{aligned}
\psi\left(\frac{\epsilon}{2}\right) & \leq \psi\left(\max \left\{\frac{\epsilon}{2}, 0,0, \frac{1}{2}\left[\frac{\epsilon}{2}+\frac{\epsilon}{2}\right]\right\}\right)-\phi\left(\max \left\{\frac{\epsilon}{2}, 0,0, \frac{1}{2}\left[\frac{\epsilon}{2}+\frac{\epsilon}{2}\right]\right\}\right) \\
& =\psi\left(\frac{\epsilon}{2}\right)-\phi\left(\frac{\epsilon}{2}\right) \\
& <\psi\left(\frac{\epsilon}{2}\right)
\end{aligned}
$$

a contradiction. Hence $\left\{y_{2 n}\right\}$ is Cauchy.

Letting $n, m \rightarrow \infty$ in

$\left|d_{p}\left(y_{2 n+1}, y_{2 m+1}\right)-d_{p}\left(y_{2 n}, y_{2 m}\right)\right| \leq d_{p}\left(y_{2 n+1}, y_{2 n}\right)+d_{p}\left(y_{2 m}, y_{2 m+1}\right)$

we get $\lim _{n, m \rightarrow \infty} d_{p}\left(y_{2 n+1}, y_{2 m+1}\right)=0$.

Hence $\left\{y_{2 n+1}\right\}$ is Cauchy.

Thus $\left\{y_{n}\right\}$ is a Cauchy sequence in $\left(X, d_{p}\right)$.

Hence, we have $\lim _{n \rightarrow \infty} d_{p}\left(y_{n}, y_{m}\right)=0$.

Now, from the definition of $d_{p}$ and from (2.3), we obtain

$$
\lim _{m \rightarrow \infty} p\left(y_{n}, y_{m}\right)=0
$$

Since $X$ is complete and $\left\{y_{n}\right\}$ is a Cauchy sequence in complete metric space $\left(X, d_{p}\right)$.

Thus

$$
\lim _{n \rightarrow \infty} d_{p}\left(y_{n}, T z\right)=0 \quad \text { for some } T z \in X
$$

Also $T$ is an ICS mapping and $\left\{y_{n}\right\}=\left\{T x_{n}\right\}$ is convergent, it follows that $\left\{x_{n}\right\}$ is convergent to some $z \in X$. i.e. $\lim _{n \rightarrow \infty} p\left(x_{n}, z\right)=p(z, z)$.

Since $\mathrm{T}$ is continuous, from above we have $\lim _{n \rightarrow \infty} p\left(T x_{n}, T z\right)=p(T z, T z)$.

By Lemma 1.4(2), we have that

$$
p(T z, T z)=\lim _{n \rightarrow \infty} p\left(T x_{n}, T z\right)=\lim _{n \rightarrow \infty} p\left(y_{n}, T z\right)=\lim _{n, m \rightarrow \infty} p\left(y_{n}, y_{m}\right)
$$

From (2.15) and (2.16), we have

$$
p(T z, T z)=0
$$

Suppose $T z \neq T F z$.

Consider

$$
\begin{aligned}
& \psi\left(p\left(T F z, y_{2 n+2}\right)\right)=\psi\left(p\left(T F z, T x_{2 n+2}\right)\right) \\
& =\psi\left(p\left(T F z, T G x_{2 n+1}\right)\right) \\
& \leq \psi \max \left(\left\{\begin{array}{c}
p\left(T z, T x_{2 n+1}\right), p(T z, T F z), p\left(T x_{2 n+1}, T x_{2 n+2}\right), \\
\frac{1}{2}\left[p\left(T z, T x_{2 n+2}\right)+p\left(T x_{2 n+1}, T F z\right)\right]
\end{array}\right\}\right) \\
& -\phi \max \left(\left\{\begin{array}{c}
p\left(T z, T x_{2 n+1}\right), p(T z, T F z), p\left(T x_{2 n+1}, T x_{2 n+2}\right), \\
\frac{1}{2}\left[p\left(T z, T x_{2 n+2}\right)+p\left(T x_{2 n+1}, T F z\right)\right]
\end{array}\right\}\right) \\
& \leq \psi \max \left(\left\{\begin{array}{c}
p\left(T z, y_{2 n+1}\right), p(T z, T F z), p\left(y_{2 n+1}, y_{2 n+2}\right), \\
\frac{1}{2}\left[p\left(T z, y_{2 n+2}\right)+p\left(y_{2 n+1}, T F z\right]\right.
\end{array}\right\}\right) \\
& -\phi \max \left(\left\{\begin{array}{c}
p\left(T z, y_{2 n+1}\right), p(T z, T F z), p\left(y_{2 n+1}, y_{2 n+2}\right), \\
\frac{1}{2}\left[p\left(T z, y_{2 n+2}\right)+p\left(y_{2 n+1}, T F z\right]\right.
\end{array}\right\}\right)
\end{aligned}
$$

Letting $n \rightarrow \infty$ and using Lemma 1.5 and (2.17), we get

$$
\begin{aligned}
\psi(p(T F z, T z)) \leq & \psi \max \left(\left\{p(T z, T z), p(T z, T F z), 0, \frac{1}{2}[p(T z, T z)+p(T z, T F z]\}\right)\right. \\
& -\phi \max \left(\left\{p(T z, T z), p(T z, T F z), 0, \frac{1}{2}[p(T z, T z)+p(T z, T F z]\}\right)\right. \\
= & \psi(p(T z, T F z))-\phi(p(T z, T F z)) \\
< & \psi(p(T z, T F z)), \text { since } \phi(t)>0 \text { for } t>0 .
\end{aligned}
$$

It is a contradiction. Hence $T F z=T z$.

Since $T$ is injective, we have $F z=z$.

As in case(a), $z$ is the common fixed point of $F$ and $G$.

Example 2.2 Let $X=[0,1]$ and $p(x, y)=\max \{x, y\}$ for all $x, y \in X$. Then $(X, p)$ is a complete partial metric space. Let $T: X \rightarrow X$ and $F, G: X \rightarrow X$ defined by $T(x)=\frac{x}{2}, F(x)=\frac{x}{2 x+4}$ and $G(x)=\frac{x^{2}}{4 x+2}$. Then it is clear that $T$ is an ICS mapping. Define $\psi \in \Psi, \phi \in \Phi$ by $\psi(t)=t$ and $\phi(t)=\frac{t}{2}$. 
Also

$$
\begin{aligned}
& \psi(p(T F x, T G y))=\max \left\{\frac{F x}{2}, \frac{G y}{2}\right\} \\
& =\frac{1}{2} \max \left\{\frac{x}{2 x+4}, \frac{y^{2}}{4 y+2}\right\} \\
& =\frac{1}{4} \max \left\{\frac{x}{x+2}, \frac{y^{2}}{y+\frac{1}{2}}\right\} \\
& \leq \frac{1}{4} \max \{x, y\} \\
& =\frac{1}{2} \max \left\{\frac{x}{2}, \frac{y}{2}\right\} \\
& =\frac{1}{2} p(T x, T y) \\
& \leq \frac{1}{2} \max \left\{\begin{array}{c}
p(T x, T y), p(T x, T F x), p(T y, T G y), \\
\frac{1}{2}[p(T x, T G y)+p(T y, T F x)]
\end{array}\right\} \\
& =\psi\left(\max \left\{\begin{array}{c}
p(T x, T y), p(T x, T F x), p(T y, T G y), \\
\frac{1}{2}[p(T x, T G y)+p(T y, T F x)]
\end{array}\right\}\right) \\
& -\phi\left(\max \left\{\begin{array}{c}
p(T x, T y), p(T x, T F x), p(T y, T G y), \\
\frac{1}{2}[p(T x, T G y)+p(T y, T F x)]
\end{array}\right\}\right)
\end{aligned}
$$

Clearly 0 is unique common fixed point of $F$ and $G$.

Corollary 2.3 Let $(X, p)$ be complete partial metric space and $T: X \rightarrow X$ be an ICS mapping and $F, G: X \rightarrow X$ be satisfying

$$
p(T F x, T G y) \leq \varphi\left(\max \left\{\begin{array}{c}
p(T x, T y), p(T x, T F x), p(T y, T G y) \\
\frac{1}{2}[p(T x, T G y)+p(T y, T F x)]
\end{array}\right\}\right)
$$

$\forall x, y \in X$, where $\varphi:[0, \infty) \rightarrow[0, \infty)$ is continous function with $\varphi(t)<t$ for $t>0$.

Then $F$ and $G$ have a unique common fixed point in $X$.

It follows from Theorem 2.1 if we put $\psi(t)=t$ and $\phi(t)=t-\varphi(t)$ in Theorem 2.1.

If we take $F=G$ in Corollary 2.3, we get

Corollary 2.4 Let $(X, p)$ be complete partial metric space and $T: X \rightarrow X$ be an ICS mapping and $F, G: X \rightarrow X$ be satisfying

$$
p(T F x, T F y) \leq \varphi\left(\max \left\{\begin{array}{c}
p(T x, T y), p(T x, T F x), p(T y, T F y) \\
\frac{1}{2}[p(T x, T F y)+p(T y, T F x)]
\end{array}\right\}\right)
$$

$\forall x, y \in X$, where $\varphi:[0, \infty) \rightarrow[0, \infty)$ is continous function with $\varphi(t)<t$ for $t>0$.

Then $F$ has a unique fixed point in $X$.

Example 2.5 Let $X=[0,1]$ and $p(x, y)=\max \{x, y\}$ for all $x, y \in X$. Then $(X, p)$ is a complete partial metric space. Let $T: X \rightarrow X$ ddefined by $T(x)=x$, it is clearly $T$ is an ICS mapping and $F: X \rightarrow X$ by $F(x)=\frac{x}{2 x+3}$ and $\varphi(t)=\frac{t}{2}$.

Also

$$
\begin{aligned}
p(T F x, T F y) & =\max \left\{\frac{x}{2 x+3}, \frac{y}{2 y+3}\right\} \\
& =\frac{1}{2} \max \left\{\frac{x}{x+\frac{3}{2}}, \frac{y}{y+\frac{3}{2}}\right\} \\
& \leq \frac{1}{2} \max \{x, y\} \\
& =\frac{1}{2} p(T x, T y) \\
& \leq \frac{1}{2} \max \left\{\begin{array}{c}
p(T x, T y), p(T x, T F x), p(T y, T F y), \\
\frac{1}{2}[p(T x, T F y)+p(T y, T F x)]
\end{array}\right\} \\
& =\varphi\left(\max \left\{\begin{array}{c}
p(T x, T y), p(T x, T F x), p(T y, T F y), \\
\frac{1}{2}[p(T x, T F y)+p(T y, T F x)]
\end{array}\right\}\right)
\end{aligned}
$$

Clearly 0 is unique fixed point of $F$.

\section{REFERENCES}

[1] T. Abdeljawad, E. Karapinar, K. Tas, Existence and uniqueness of a common fixed point on partial metric spaces, Appl. Math. Lett. 24 (11),1894-1899(2011) (2011).

[2] I. Altun, F. Sola and H. Simsek, Generalized contractions on partial metric spaces, Topology and its Applications. 157 (18) (2010) 2778-2785.

[3] I. Altun and A. Erduran, Fixed point theorems for monotone mappings on partial metric spaces, Fixed Point Theory and Applications, Volume 2011, Article ID 508730, 10 pages, doi:10.1155/2011/508730. 
[4] H. Aydi, Fixed point results for weakly contractive mappings in ordered partial metric spaces, Journal of Advanced Mathematical and Studies, Volume 4, Number 2, (2011).

[5] K.P.chi, On a fixed point theorem for certain class of maps satisfying a contractive condition depended on an another function, Lobachevskii J. math., 30(4), 2009, 289 - 291.

[6] D. Ilić, V. Pavlović, V. Rakočević, Some new extensions of Banach's contraction principle to partial metric spaces, Appl. Math. Letters, doi:10.1016/j.aml.2011.02.025.

[7] E. Karapınar, I. M. Erhan, Fixed point theorems for operators on partial metric spaces, Applied Mathematics Letters 24 (11),1900-1904 (2011), 10.1016/j.aml.2011.05.013.

[8] Karapinar, E.: Weak $\phi$-contraction on partial contraction and existence of fixed points in partially ordered sets, Mathematica Aeterna, 1(4)237-244(2011).

[9] Karapınar, E.: Generalizations of Caristi Kirk's Theorem on Partial metric Spaces, Fixed Point Theory and. Appl. 2011:4, doi:10.1186/1687-1812-2011-4

[10] S.G. Matthews. Partial metric topology. Research Report 212. Dept. of Computer Science. University of Warwick, 1992.

[11] S.G. Matthews, Partial metric topology, in Proceedings of the 8th Summer Conference on General Topology and Applications, vol. 728, pp. 183-197, Annals of the New York Academy of Sciences, 1994.

[12] M.Schellekens, The Smtth comletion: a common foundation for denotational semantics and complexity analysis, Electronic Notes in Theoretical Computer Science, vol 1, 1995, 535 - 556.

[13] P.Waszkiewicz, Quantitative continuous domains, Applied Categorical Structures, vol 11, no. 1, 2003,41 - 67.

[14] P.Waszkiewicz, Partial metrizebility of continuous posets, Mathematical Structures in Computer Sciences, vol 16, no. 2, 2006, $359-372$.

[15] R. Heckmann, Approximation of metric spaces by partial metric spaces, Appl. Categ. Structures,no.1-2, 7, 1999, 71-83.

[16] R. Kopperman, S.G. Matthews, and H. Pajoohesh: What do partial metrics represent?, Spatial representation: discrete vs. continuous computational models, Dagstuhl Seminar Proceedings, No. 04351, Internationales Begegnungs- und Forschungszentrum fr Informatik (IBFI), Schloss Dagstuhl, Germany, (2005). MR 2005j:54007

[17] S.J. ONeill: Two topologies are better than one, Tech. report, University of Warwick, Coventry, UK, http://www.dcs.warwick.ac.uk/reports/283.html, (1995).

[18] H.P.A. Künzi, H. Pajoohesh, and M.P. Schellekens: Partial quasi-metrics, Theoret. Comput. Sci. 365 no.3 (2006) 237-246. MR 2007f:54048

[19] S. Romaguera and M. Schellekens: Weightable quasi-metric semigroup and semilattices, Electronic Notes of Theoretical computer science, Proceedings of MFCSIT, 40, Elsevier, (2003).

[20] M.P. Schellekens: A characterization of partial metrizability: domains are quantifiable, Topology in computer science (Schlo Dagstuhl, 2000), Theoretical Computer Science 305 no. 1-3 (2003) 409-432. MR 2004i:54037

[21] S. Romaguera, A Kirk type characterization of completeness for partial metric spaces, Fixed Point Theory and Applications, Volume 2010, Article ID 493298, 6 pages, 2010.

[22] O. Valero, On Banach fixed point theorems for partial metric spaces, Applied General Topology. 6 (2) (2005) 229-240.

[23] S. Oltra and O. Valero, Banach's fixed point theorem for partial metric spaces, Rendiconti dell'Istituto di Matematica dell'Università di Trieste. 36 (1-2) (2004) 17-26. 